\title{
A new species of the multistriatum species-group of Simulium (Simulium) (Diptera: Simuliidae) from Northern Thailand
}

\author{
Hiroyuki Takaoka ${ }^{1}$ and Wej Choochote ${ }^{2}$ \\ Accepted 27, March, 2007
}

\begin{abstract}
Simulium (Simulium) chanyae sp. nov. is described based on two female adult specimens collected in Northern Thailand. This new species is assigned to the multistriatum species-group of the subgenus Simulium (Simulium), and is easily distinguished from most of the other known species of this species-group by the yellowish legs.
\end{abstract}

Key words. Simulium, black fly, Simuliidae, Thailand, new species

During recent surveys on adult black flies in Doi Suthep-Pui National Park, Chiang Mai Province, Northern Thailand, we collected a new species which is assigned to the multistriatum species-group of the subgenus Simulium (Simulium) Latreille s. str., redefined by Takaoka (2003). This new species is distinguished from all the known species of the same species-group (including eight species from Thailand) by the color of the legs, especially mid basitarsus (Crosskey and Howard, 2004; Takaoka and Choochote, 2005a, b, c; Takaoka and Kuvangkadilok, 1999).

This new species is described herein as the ninth species of the multistriatum species-group in Thailand.

The terms for morphological features used here follow those of Takaoka (2003). Holotype and paratype specimens of the new species are deposited at the Department of Infectious Disease Control, Faculty of Medicine, Oita University, Oita, Japan.

\section{Simulium (Simulium) chanyae sp. nov.}

DESCRIPTION. Female. Body length $2.9 \mathrm{~mm}(3.0$ $\mathrm{mm}$ in paratype). Head. Narrower than thorax. Frons dark brown to brownish-black, shiny, with several dark stout hairs along lateral margins; frontal ratio 1.41:1.00:1.65; frons-head ratio 1.0:4.3. Fronto-ocular area (Fig. 1A) well developed, short, directed laterally and somewhat upwardly. Clypeus dark brown to brownish-black, thickly white pruinose, slightly shiny at a certain angle of light, moderately covered with dark stout hairs except upper $2 / 3$ widely bare medially. Labrum 0.68 times as long as clypeus. Antenna composed of scape, pedicel, and 9 flagellomeres, yellow on scape, pedicel, and 1st to 3rd flagellomeres and light to medium brown on other flagellomeres when viewed from the front (in paratype, antenna more extensively yellow, i. e., scape, pedicel and 1st to 5th flagellomeres yellow and rest medium brown when viewed from the front); 1st flagellomere 1.62 times as long as the 2nd one. Maxillary palp with 5 segments, light to dark brown, proportional lengths of 3rd, 4th, and 5th segments 1.00:1.07:2.14; 3rd segment (Fig. 1B) of normal size, with large oblong sensory vesicle (0.40 times as long as 3rd segment) having medium-sized opening submedially. Maxillary lacinia with 11 inner and 12 outer teeth (12 inner and 13 outer teeth in paratype). Mandible with 22 inner and 12 outer teeth (26 inner and 13 outer teeth in paratype). Cibarium (Fig. 1C) with blunt median projection on posterior margin and without any minute processes near base of median projection. Thorax. Scutum dark brown to brownish-black, shiny, densely covered with whitish-yellow recumbent short hairs interspersed with several dark long upright hairs on prescutellar area; when illuminated in front and viewed dorsally, scutum thickly white pruinose, with 5 non-pruinose longitudinal vittae (i. e., 1 median vitta of moderate width, 2 submedian vittae somewhat wider than the median one and 2 sublateral vittae much wider than the 2 submedian ones), all vittae united with broad transverse band on prescutellar area; scutum having a reversed color pattern when illuminated from behind. Scutellum medium to dark brown, covered with dark upright long hairs as well as whitish-yellow short hairs. Postnotum dark brown to brownish-black, shiny, gray pruinose, bare. Pleural membrane bare. Katepisternum longer than deep, bare. Legs. Foreleg: coxa and trochanter 
yellowish-white; femur (Fig. 1D) yellow except apical cap dark yellow to light brown; tibia white except apical cap dark brown; tibia with white large sheen on outer surface; tarsus brownish-black, with moderate dorsal hair crest; basitarsus greatly dilated, 4.4 (4.5 in paratype) times as long as its greatest width. Midleg: coxa brownish-black; trochanter yellow; femur (Fig. 1E) yellow on basal 1/3 or a little more, light brown on rest (though the border is not well defined) except apical cap medium brown (femur yellow with apical cap light brown in paratype); tibia white to yellowish-white except apical cap light to medium brown; tibia with white large sheen on posterior surface; tarsus medium to dark brown except basal 2/3 of basitarsus yellowish-white (Fig. 1 F). Hind leg: coxa dark brown; trochanter yellowish-white; femur (Fig. 1G) yellowish-white basally, becoming yellow up to a little less than basal $1 / 2$, then dark yellow (light brown medially in paratype) on rest except apical cap medium to dark brown; femur light brown on posterior surface of apical 2/5; tibia white to yellowish-white on a little less than basal $4 / 5$ (on a little less than basal $3 / 4$ in paratype), medium to dark brown on rest; tibia with white large sheen on posterior surface; tarsus dark brown except a little more than basal $3 / 5$ of basitarsus, and a little less than basal 1/2 of 2nd segment yellowish-white (Fig. 1H); basitarsus (Fig. 1H) nearly parallel-sided, 5.71 (5.76 in paratype) times as long as wide, and 0.77 and 0.63 times as wide as greatest width of hind tibia and femur, respectively; calcipala (Fig. 1 $\mathrm{H})$ moderately developed, 0.81 times as long as its basal width, and 0.41 times as wide as greatest width of basitarsus; pedisulcus (Fig. 1H) well developed at basal 1/3 of 2 nd tarsal segment. All tarsal claws simple. Wing. Length 2.4 mm. Costa with dark spinules and hairs; subcosta fully haired up to or near apex; basal section of radial vein bare; $\mathrm{R}_{1}$ with dark spinules and hairs; $\mathrm{R}_{2}$ with hairs; hair tuft on stem vein dark brown; basal cell absent. Abdomen. Basal scale dark yellow to light brown, with fringe of pale hairs. Dorsal surface of abdomen dark brown to brownish-black except basal 3/5 of 2nd segment yellowish-white, moderately covered with medium-long to long dark stout hairs interspersed with short to medium-long ocherous hairs on posterior segments; tergite 2 shiny, white iridescent when illuminated, and tergites 6-8 shiny. Ventral surface of segment 7 with large weakly sclerotized sternal plate medially. Genitalia. Sternite 8 (Fig. 1I) with about 21-25 dark medium-long to long stout hairs on each lateral surface. Ovipositor valves (Fig. 1I) tongue-like, rounded posteromedially, moderately separated from each other, membranous, moderately covered with microsetae and 2-5 pale or dark medium-long hairs, except portion along inner margin widely bare, and so thin and transparent that it is difficult to discern inner margins. Genital fork (Fig. 1J) of inverted-Y form, with narrow well-sclerotized stem; arms of moderate width, each with distinct projection directed anterodorsally. Paraproct (Fig. 1K,L) strongly sclerotized on outer surface but unsclerotized on anterior and inner surfaces except small portion sclerotized, moderately protruding ventrally beyond cercus, with numerous stout hairs on lateral and ventral surfaces, and with 5 or 6 short sensilla on inner surface. Cercus (Fig. 1K,L) short, 0.45 times as long as wide, with numerous stout hairs, and rounded posteriorly when viewed laterally. Spermatheca (Fig. 1M) nearly ovoid, well sclerotized except small portion of junction to duct unsclerotized, with very weakly defined reticulate patterns on its surface, and with internal setae; accessory ducts subequal in thickness to each other, and subequal to, or slightly thicker than, major duct.

Male, pupa and mature larva. Unknown.

TYPE SPECIMENS. Holotype female, collected while attracted to a human, at Ban Mong Doi Pui Village (altitude 1,460 m), Doi Suthep-Pui National Park, Chiang Mai Province, Northern Thailand, 8.X.2005, by W. Choochote. Paratype: 1 female, same data as those of the holotype, except the date, 9.X.2005.

ETYMOLOGY. The specific name chanyae honors Mrs. Chanya Choochote, who has been supporting Associate Professor Wej Choochote for many years.

REMARKS. According to the key (Takaoka, 2003), $S$. $(S$.$) chanyae sp. nov. is assigned to the multistriatum$ species-group of the subgenus Simulium s. str. on the basis of the scutum with five longitudinal vittae composed of a median, 2 submedian and 2 sublateral vittae, simple claws and the ovipositor valves being very thin and bare widely along the inner margins (Fig. 1I).

Simulium $(S$. ) chanyae sp. nov. seems to be most closely related to $S$. (S.) novolineatum Puri, 1933 from India (Puri, 1932; 1933) in view of the female femora extensively yellowish except their apical caps darkened, but is distinguished from the latter species by the basitarsus of the middle leg which is yellowish-white on the basal 2/3 (Fig. 1 F) in this new species but yellowish on the basal $1 / 2$ in $S$. (S.) novolineatum, and also by the spermatheca which is ovoidal (Fig. 1M) in this new species but globular in $S$. (S.) novolineatum.

Among the other known species of the multistriatum species-group, S. (S.) multistriatum Rubtsov, 1947 from Tazhikistan and Uzbekistan, S. (S.) triglobus Takaoka and Kuvangkadilok, 1999 from Thailand, and S. (S.) xanthogastrum Rubtsov, 1951 from Tazhikistan seem somewhat re- 

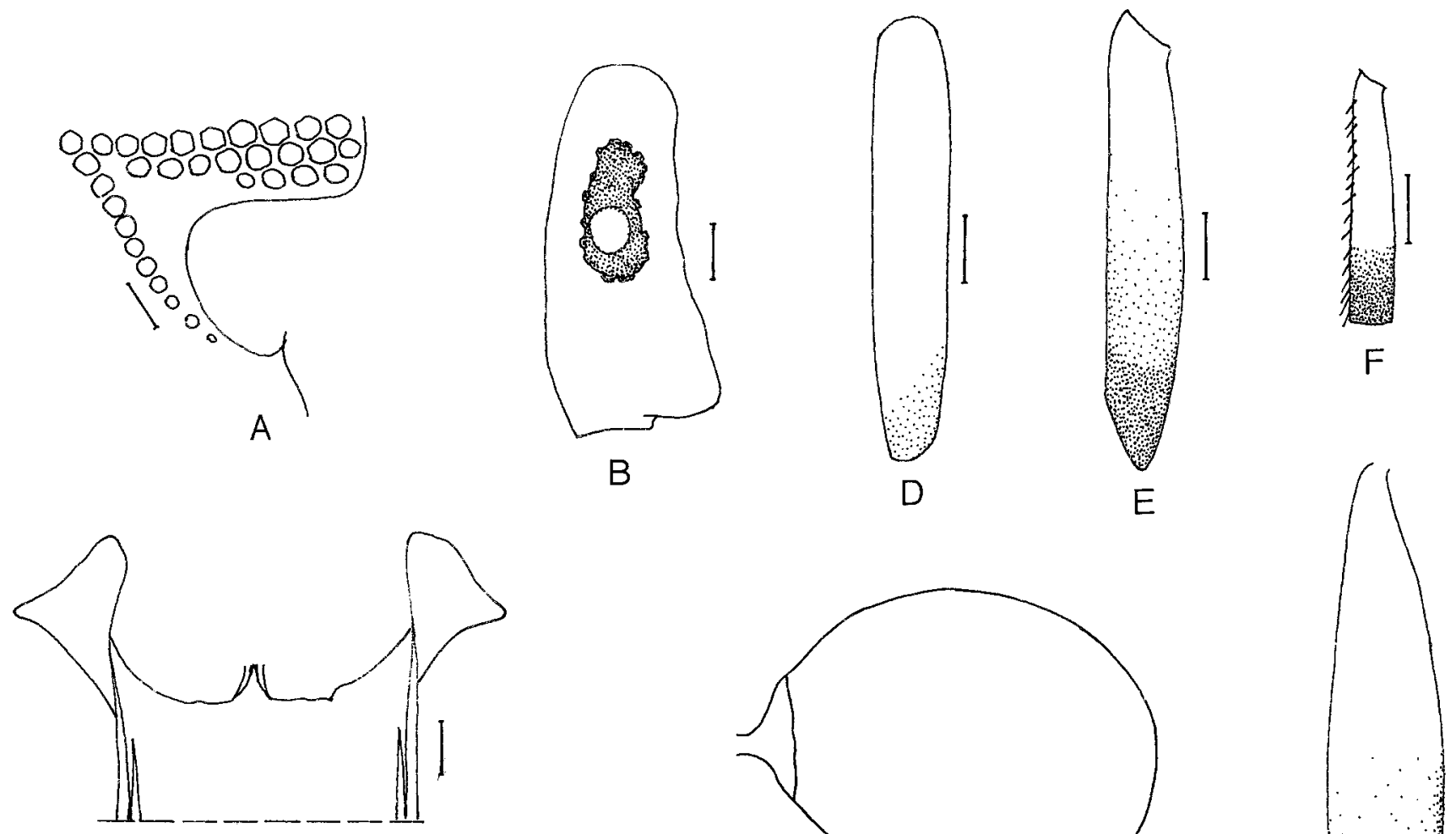

$\mathrm{D}$

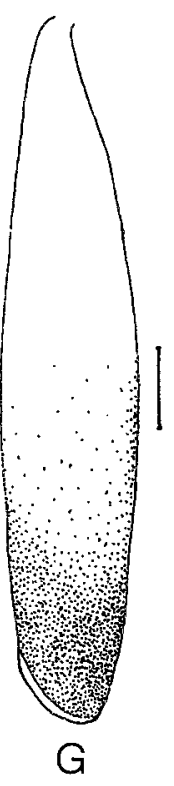

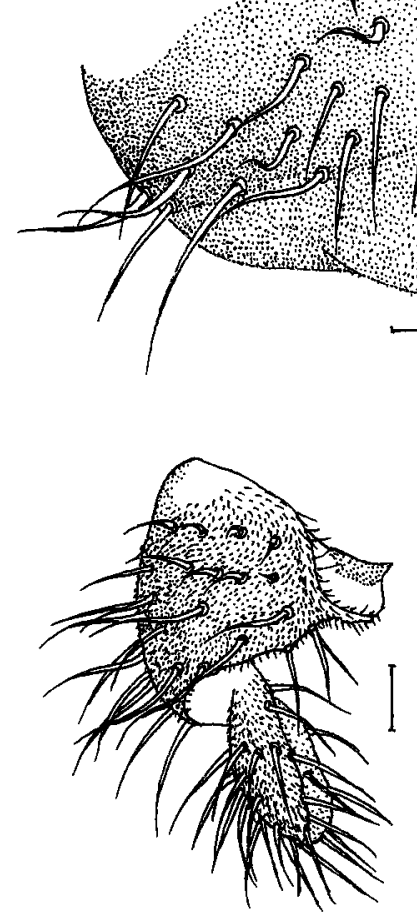

$\mathrm{K}$

C
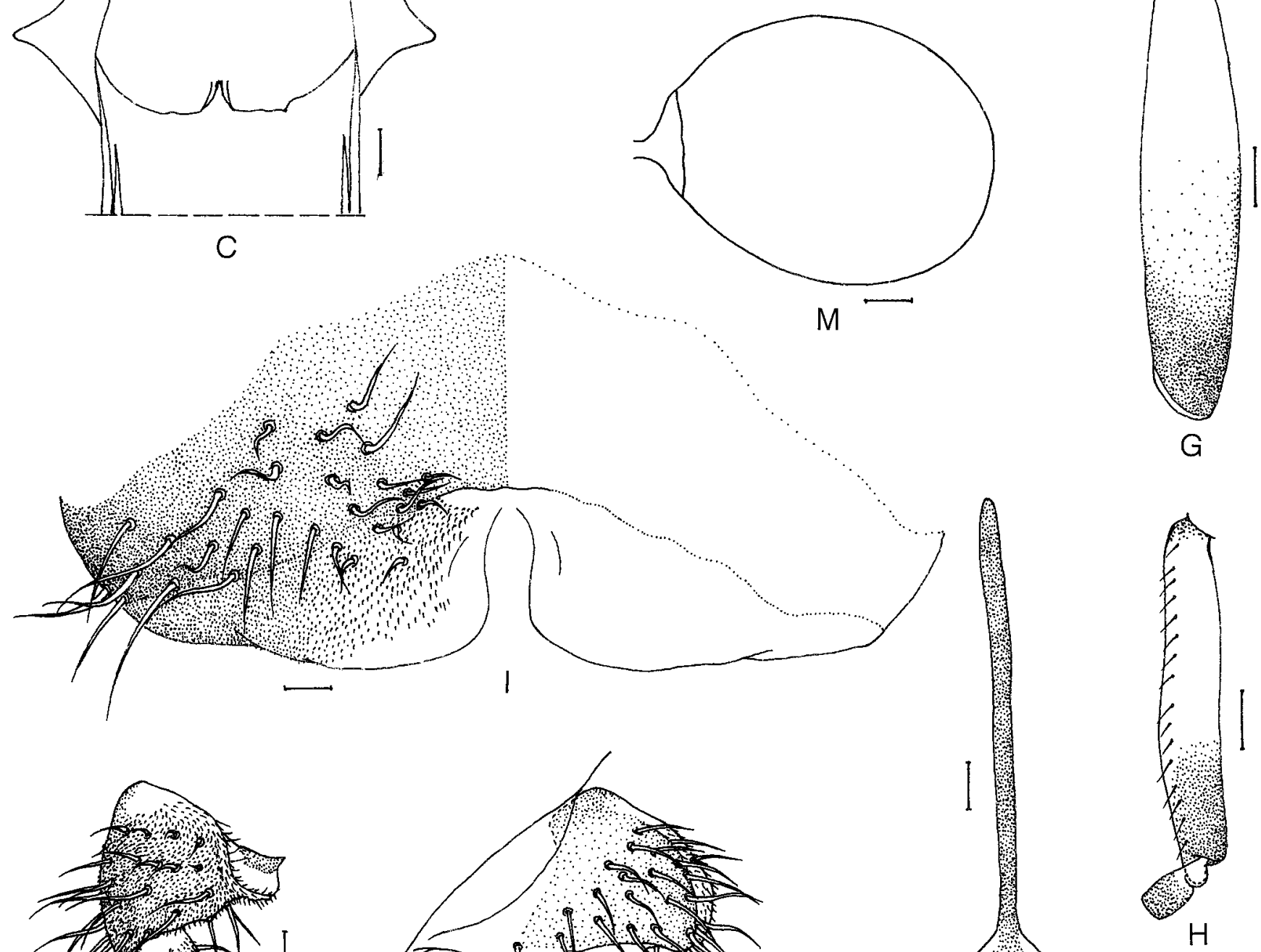

Fig. 1. Female of Simulium (Simulium) chanyae sp. nov. A, fronto-ocular area (right side); B, 3rd segment of maxillary palp with sensory vesicle (right side; front view); C, cibarium; D, fore femur (outer view); E, mid femur (outer view); F, mid basitarsus (outer view); G, hind femur (outer view); H, hind basitarsus and 2nd tarsal segment (outer view); I, 8th sternite and ovipositor valves in situ (ventral view); J, genital fork (ventral view); K and L, paraprocts and cerci in situ (right side; K, ventral view; L, lateral view); M, spermatheca. Scale bars. $0.1 \mathrm{~mm}$ for D-H; $0.03 \mathrm{~mm}$ for A; $0.02 \mathrm{~mm}$ for B,C and I-M. 
lated to this new species in view of the yellowish female legs, but all their femora are entirely yellowish, according to Rubtsov (1956) and Takaoka and Kuvangkadilok (1999).

It is noteworthy that $S$. (S.) sp. collected from Myanmar, tentatively identified by us as $S$. ( $S$. ) chanyae based on the female morphological characteristics, has a simple wallpocket-shaped cocoon without lateral windows (Takaoka, unpublished data). Among the known species of the multistriatum species-group, $S$. (S.) malayense is the only species known to have such a simple cocoon (Takaoka and Davies, 1995), but its other pupal characteristics including the head and thoracic integuments almost bare, and the gill filaments diverging at a right angle when viewed laterally, are different from those of $S$. (S.) chanyae. Unlike $S$. (S.) chanyae sp. nov., the female femora of $S$. (S.) malayense are dark (Takaoka and Davies, 1995).

\section{ACKNOWLEDGEMENTS}

This study was financially supported by a Grant-in-Aid for Overseas Research from the Japan Society for the Promotion of Science (No. 18406011).

\section{REFERENCES}

Crosskey, R.W. and Howard, T.M. 2004. A Revised Taxonomic and Geographical Inventory of World Blackflies (Diptera: Simuliidae). 78 pp., The Natural History Museum, London.

Puri, I.M.1932. Studies on Indian Simuliidae. Part III. Descrip- tions of males, females and pupae of $S$. griseifrons Brunetti (1911) and of four new species with striped thorax. Ind. J. Med. Res., 19: 1125-1143.

Puri, I.M.1933. Studies on Indian Simuliidae. Part VII. Descriptions of larva, pupa and female of Simulium nodosum sp. nov., with an appendix dealing with $S$. novolineatum nov. nom. (=S. lineatum Puri). Ind. J. Med. Res., 20: 813-817.

Rubtsov, I.A. 1956. Blackflies (Simuliidae)[Moshki (sem. Simuliidae)]. Fauna of the USSR. 859 pp., New Series No. 64, Insects, Diptera, 6 (6). Akademiia Nauk SSSR, Leningrad [=St. Petersburg], Russia. (In Russian; English translation: 1990. Blackflies (Simuliidae). 1,042 pp., 2nd Ed. Fauna of the USSR. Diptera, 6 (6). E.J.Brill, Leiden]

Takaoka, H. 2003. The Black Flies (Diptera: Simuliidae) of Sulawesi, Maluku and Irian Jaya. xxii + 581 pp., Kyushu University Press, Fukuoka.

Takaoka, H. and Choochote, W. 2005a. A new species of Simulium (Simulium) (Diptera: Simuliidae) from Thailand. Med. Entomol. Zool., 56: 43-47.

Takaoka, H. and Choochote, W. 2005b. Two new species of Simulium Latreille (Diptera: Simuliidae) from northwestern Thailand. Med. Entomol. Zool., 56: 123-133.

Takaoka, H. and Choochote, W. 2005c. Two new species of black flies (Diptera: Simuliidae) from northern Thailand. Med. Entomol. Zool., 56: 319-334.

Takaoka, H. and Davies, D.M. 1995. The Black Flies (Diptera: Simuliidae) of West Malaysia.viii + 175 pp., Kyushu University Press, Fukuoka.

Takaoka, H. and Kuvangkadilok, C. 1999. Four new species of black flies (Diptera: Simuliidae) from Thailand. Jpn. J. Trop. Med. Hyg., 27: 497-509. 